\title{
Choice of High Occupancy/Toll Lanes Under Alternative Pricing Strategies
}

\author{
Michael Janson and David Levinson
}

\begin{abstract}
High Occupancy/Toll (HOT) Lanes typically vary tolls charged to single occupant vehicles (SOVs), with the toll increasing during congested periods. The toll is usually tied to time of day or to the density of vehicles in the HOT lane. The purpose of raising the toll with congestion is to discourage demand sufficiently to maintain travel speeds in the HOT lane. However, Janson and Levinson (2014) demonstrated that the HOT toll may act as a signal of downstream congestion (in both general purpose (GP) and HOT lanes), causing an increase in demand for the HOT lane, at least at lower prices. This paper develops a model of lane choice to evaluate alternative HOT lane pricing strategies, including the use of GP density, to more accurately reflect the value of the HOT lane. In addition, the paper explores the potential effect these strategies would have on the HOT lane vehicle share through a partial equilibrium analysis. This analysis demonstrates the change in demand elasticity with price, showing the point at which drivers switch from a positive to negative elasticity.
\end{abstract}

Total Words $=3403+250 * 14(6$ Figures $\& 8$ Tables $)=7403$ 


\section{Introduction}

While toll roads originated to finance infrastructure and earn profits for their owners, it has been long understood that tolls could also be used to manage traffic, as higher tolls tend to deter usage (Dupuit, 1844; Vickrey, 1969; Pahaut and Sikow, 2006; Yang and Huang, 2005; Small et al., 2012). The construction of High Occupancy Vehicle (HOV) lanes, beginning with the Shirley Highway in 1969, launched as a bus-only lane and subsequently opened to carpools in 1973 during the energy crisis, aimed to provide an incentive for travelers to carpool to increase average auto occupancy and person-throughput on roadways, and thus lower energy use. In general however, such facilities were inefficient, either the occupancy threshold was too low, and the lanes carried as many vehicles as general purpose lanes, or it was too high, and the lane was underutilized, leaving vehicle capacity unused (Dahlgren, 1998, 2002).

In recent years many HOV lanes in the United States have been converted to High Occupancy/Toll (HOT) lanes, opening the lanes which were underused to additional toll-paying traffic. The toll serves to raise revenue to cover operating costs and to regulate the demand of SOVs. HOT lanes across the United States use different methods for determining the toll; however, all such facilities raise the toll during more congested periods. The theory is, a higher toll price discourages demand and is used to maintain a high level of service in the HOT lane(s). In practice, however, Janson and Levinson (2014) showed that a higher price may act as a signal of downstream congestion (in both the general purpose (GP) and HOT lanes), causing demand for the HOT lane to increase, at least to a point. Evaluations of alternative pricing schemes have been limited (Gardner et al., 2014).

This paper develops a model to predict HOT lane choice on Minnesota's well-studied MnPASS lanes (Halvorson and Buckeye, 2006; Munnich and Buckeye, 2007; Tilahun and Levinson, 2009; Goodall and Smith, 2010; Cao et al., 2012; Buckeye, 2012). It then develops some alternative HOT pricing strategies. These alternative strategies are tested using a partial equilibrium analysis. This analysis uses the calibrated HOT lane choice model to predict the HOT lane share at various prices and estimate demand elasticity to price.

\section{Pricing on MnPASS Lanes}

The MnPASS lanes in Minneapolis were an early application of dynamic toll pricing in the United States, opening in 2004. With several exceptions, the general operating hours are from 6:00-10:00 and 15:00-19:00. Prices during operation range from a minimum of $\$ 0.25$ to a maximum $\$ 8.00$. Each route (I-394, I-35W, and I-35E) is divided into multiple sections with prices posted for use of each segment. The maximum price applies to use of each section individually, as well as use of all sections. 
Prices are adjusted every three minutes based on density levels measured in the MnPASS lanes only. Traffic levels in the general purpose (GP) lanes do not directly influence price. Loop detector counts are taken every 30 seconds and used to calculate the density in the MnPASS virtual toll collection plazas along the corridor. Density measurements are averaged over the most recently completed 6 minute period in order to smooth out fluctuations and based only on downstream congestion. Price is dictated by the magnitude of density as well as the change in density over the previous 6 minutes. A rise in density creates an increase in price.

Table 1 displays the pricing plan in effect for most of the history of the express lanes, and the plan in effect when the empirical analyses described below were conducted. It remained in effect until 2015, when it was replaced by a continuous function (Harlow, 2015). Both of these approaches regulate the price based on traffic density level. Minimums and maximums for a given level of service must be maintained. The table also indicates the changes in price caused by a change in density.

Table 1: Pricing Plan for Normal Operation of MnPASS Lanes (both I-35W and I-394) at Time of Study

\begin{tabular}{|c|c|c|c|c|c|c|}
\hline Level of Service & Min K & $\operatorname{Max} \mathrm{K}$ & Min Rate $(\$)$ & Default Rate $(\$)$ & Max Rate $(\$)$ & \\
\hline $\mathrm{A}$ & 0 & 11 & 0.25 & 0.25 & 0.50 & \\
\hline B & 12 & 18 & 0.50 & 0.50 & 1.50 & \\
\hline $\mathrm{C}$ & 19 & 31 & 1.50 & 1.50 & 2.50 & \\
\hline $\mathrm{D}$ & 32 & 42 & 2.50 & 3.00 & 3.50 & \\
\hline $\mathrm{E}$ & 43 & 49 & 3.50 & 5.00 & 5.00 & \\
\hline $\mathrm{F}$ & 50 & 50 & 5.00 & 8.00 & 8.00 & \\
\hline \multicolumn{7}{|c|}{ Change in Price from Density Change } \\
\hline $\mathrm{K}$ & $\Delta 1$ & $\Delta 2$ & $\Delta 3$ & $\Delta 4$ & $\Delta 5$ & $\Delta 6$ \\
\hline $0-18$ & 0.25 & 0.25 & 0.25 & 0.25 & 0.25 & 0.25 \\
\hline $19+$ & 0.25 & 0.50 & 0.75 & 1.00 & 1.25 & 1.50 \\
\hline
\end{tabular}

\section{HOT Lane Choice}

\subsection{Methods}

The analysis uses a fixed number of SOVs with predefined commute times and locations to calibrate a lane choice model. Transponder-equipped SOVs can decide whether to use the MnPASS or GP lanes based on the toll and their expected travel time and reliability. 
The HOT lane choice model extends Carrion (2010). The binomial logit model determines the probability of a vehicle using the HOT lane based on several independent variables. These variables include estimated travel time and travel time variability for both the HOT lane and the GP lanes, as well as the posted toll price. The lane choice model applies only to SOVs equipped with transponders. SOVs not equipped with transponders are not allowed to use the MnPASS lanes. A separate subscription choice model was developed to determine which vehicles are equipped with transponders (Owen et al., 2014).

Utility of the HOT lane is described as:

$$
U=f(T, V, P, A)
$$

where:

T: Expected Travel Time The utility decreases with an increase in expected travel time, decreasing the probability of using the given lane type. Expected travel time is measured in minutes.

V: Travel Time Variability Travel time variability in this model is defined as the 90th percentile - 50th percentile to correspond with Carrion (2010). This value is calculated separately for the HOT lane and GP lanes. Like expected travel time, an increase in variability decreases the probability of using that lane. Travel time variability is measured in minutes.

P: Expected Toll Price The expected toll variable is based on the dynamic message sign posted price. The price corresponds to a user's entry and exit points. This model assumes all drivers will exit in downtown Minneapolis. Therefore, the expected toll will vary only by entry point. Toll prices are in USD. The negative sign indicates a dissuasion from higher tolls, assuming all other factors remain constant.

A: Alternative Specific Constant In this model, the ASC was defaulted to zero and adjusted if necessary in the calibration.

The lane choice model was calibrated by matching a set of simulated vehicles' HOT lane decisions to historical data. A list of vehicles was generated from trip tables provided by the Metropolitan Council. All vehicles are SOVs traveling eastbound to downtown Minneapolis on I-394 between 6:00-10:00 AM. Each vehicle has an entrance ramp and time of entry into the system. The subscription choice model from Owen et al. (2014) is first applied to filter non-transponder owning SOVs. Each vehicle experiences various travel times based on the entrance ramp and time of entry. These travel times are the basis of the expected travel time 
and travel time reliability parameters of the lane choice model. Details of the calibration steps are outlined below.

The lane choice model coefficients are adjusted using a grid search technique. Default values for the coefficients were taken from Carrion (2010), with the exception of the alternative specific constant (ASC) which was set to zero. The grid search approach involves adjusting each of the coefficients separately (travel time first, then tolls). The first coefficient is altered until the model achieves its best fit to the calibration target. This coefficient is then kept constant and the second coefficient is adjusted until the fit can no longer be improved. The ratio of expected travel time to travel time variability was kept constant due to the extensive literature research outlined in from Carrion and Levinson (2012) in determining this value.

Each vehicle builds a travel time history by experiencing MnPASS travel times along the corridor based on their entrance ramp and time of entry. All travel is along I-394 Eastbound to downtown Minneapolis. The travel times are calculated using loop detector data from each Wednesday of 2012 except holidays: July 4 and December 26. This travel history determines a vehicle's expected travel time (mean of travel history) and travel time variability (90th percentile minus 50th percentile).

In order to calibrate the lane choice model, it is necessary to determine the probability that a transponder-owning SOV will use the MnPASS lane.

Using Bayes' theorem:

$$
\operatorname{Pr}(L \mid R)=\operatorname{Pr}(R \mid L) * \operatorname{Pr}(L) / \operatorname{Pr}(R)
$$

$\operatorname{Pr}(R)$ is the probability of radio transponder ownership (from subscription choice model). $\operatorname{Pr}(L)$ represents the probability of using the HOT lane among all SOVs. $\operatorname{Pr}(R \mid L)$ is the probability of owning a transponder given use of the HOT lane. Since only SOVs are being considered, $\operatorname{Pr}(R \mid L)$ is 1 (or 100\%) assuming no illegal use of the HOT lane.

$\operatorname{Pr}(L)$ was calculated by finding the number of SOVs using the MnPASS lane and dividing by total number of vehicles using the corridor during the same time period. Total vehicle counts were gathered from loop detector data. The number of HOVs using the GP lanes is assumed to be zero. Counts of SOVs using the MnPASS lane come from transponder data which shows entry and exit plazas and entry time, along with paid toll price. By comparing the counts throughout morning peak period with the GP loop detector data, $\operatorname{Pr}(L)$ can be determined.

$\operatorname{Pr}(R)$ was calculated by correlating the subscription choice model in Owen et al. (2014) with subscription data for each transportation analysis zone (TAZ) along the corridor. Each vehicle's entrance ramp can be probabilistically correlated to surrounding TAZs. By then applying the subscription choice model to the total set of SOVs, a subset of transponder equipped SOVs is formed. This is likely a lower bound of transponder usage, since transpon- 
der owners in a TAZ are more likely to use MnPASS (or MnPASS corridor users are more likely to own a transponder) than a random traveler from a TAZ.

Due to the connection of this research to the calibrated traffic simulation, November 29, 2011 was selected for calibration of the lane choice model. It was an 'average' day with no weather or crash related problems along the MnPASS corridors.

The $\operatorname{Pr}(L)$ value from that day and $\operatorname{Pr}(R)$, result in:

$$
\operatorname{Pr}(L \mid R)=(100 \%) *(11.8 \%) /(17.3 \%)=68.1 \%
$$

Although the MnPASS toll price fluctuates based on HOT density, there is a direct correlation between the toll and the time savings the MnPASS lanes provide over the GP lanes; so the higher the toll, the greater the time savings. This correlation is observed by users and explains the positive demand elasticity to price results in Janson and Levinson (2014).

\subsection{Results}

Using average toll prices and time savings data from 2012, a log relationship was fit. The bimodal relationship of the data meant two log functions were fit, one for congestion onset and one for offset.

The relationship between price and time savings (and travel time variance) during congestion onset and offset are displayed in Table 2. The corresponding price time-savings curves are displayed in Figure 1. The price reliability curves are displayed in Figure 2.

Table 2: Price-Time Savings and Price-Reliability Regression Results

\begin{tabular}{crrrrrrrr}
\hline & \multicolumn{3}{c}{ Time Savings vs Price } & \multicolumn{4}{c}{ Time Variance Difference vs Price } \\
\hline Variable & Onset & & Offset & & Onset & & Offset & \\
Intercept & 0.5527 & $* * *$ & 1.2965 & $* * *$ & 0.9566 & $* * *$ & 1.6636 & $* * *$ \\
& $(0.05972)$ & & $(0.02912)$ & & $(0.03867)$ & & $(0.01664)$ & \\
$\ln (P)$ & 1.2587 & $* * *$ & 0.7953 & $* * *$ & 1.1413 & $* * *$ & 0.926 & $* * *$ \\
& $(0.07732)$ & $(0.03743)$ & & $(0.05006)$ & $(0.02139)$ & \\
$n$ & 40 & 40 & 40 & & 40 & \\
$r^{2}$ & 0.8923 & 0.913 & 0.942 & & 0.9657 & \\
\hline
\end{tabular}

(Standard error in parentheses)

Significance *0.05, **0.01, *** 0.001

Time Savings and Time Variance Difference in minutes are the dependent variables, price in USD is the independent variable 
Figure 1: Price-Time Savings Log Model

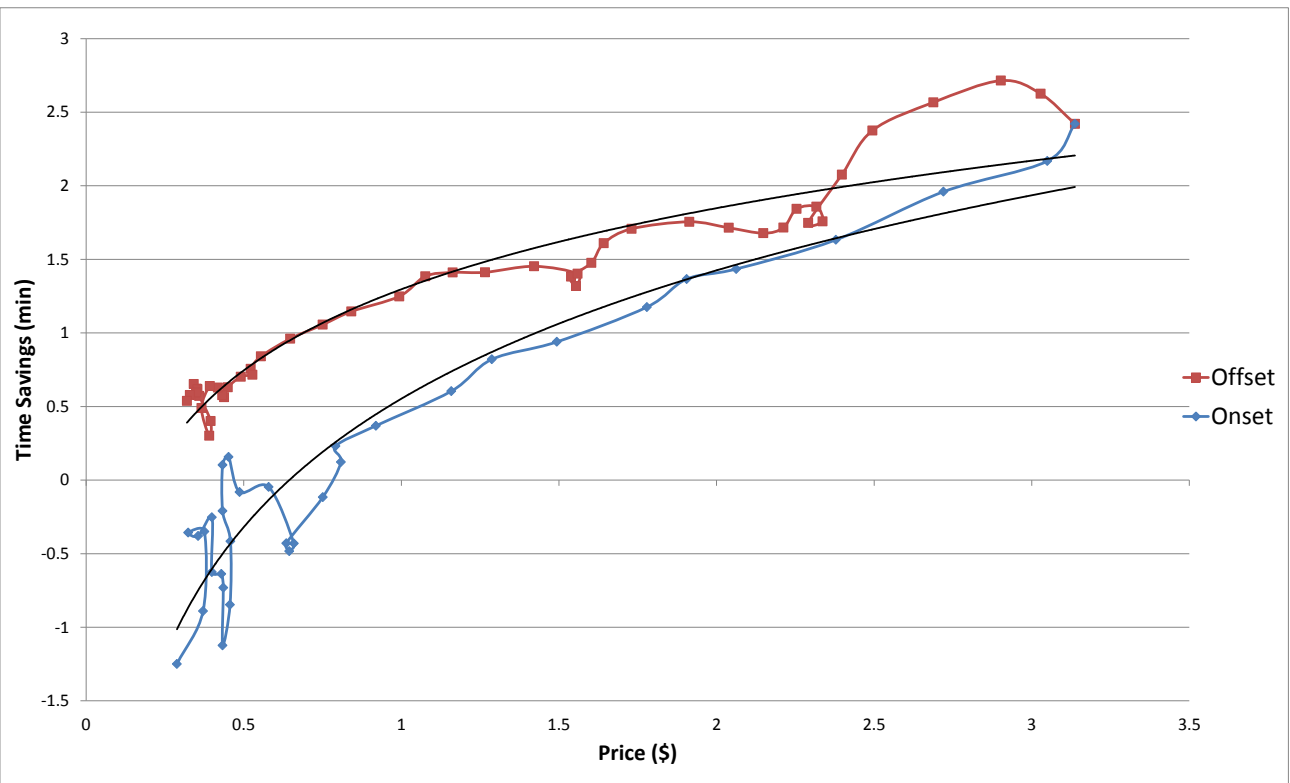

$\Delta T_{\text {onset }}=1.2587 \ln (P)+0.5527\left(r^{2}=0.8923\right)$

$\Delta T_{\text {offset }}=0.7953 \ln (P)+1.2965\left(r^{2}=0.913\right)$

where $\Delta T$ is travel time savings in minutes and $P$ is price in USD

Figure 2: Price-Reliability Model

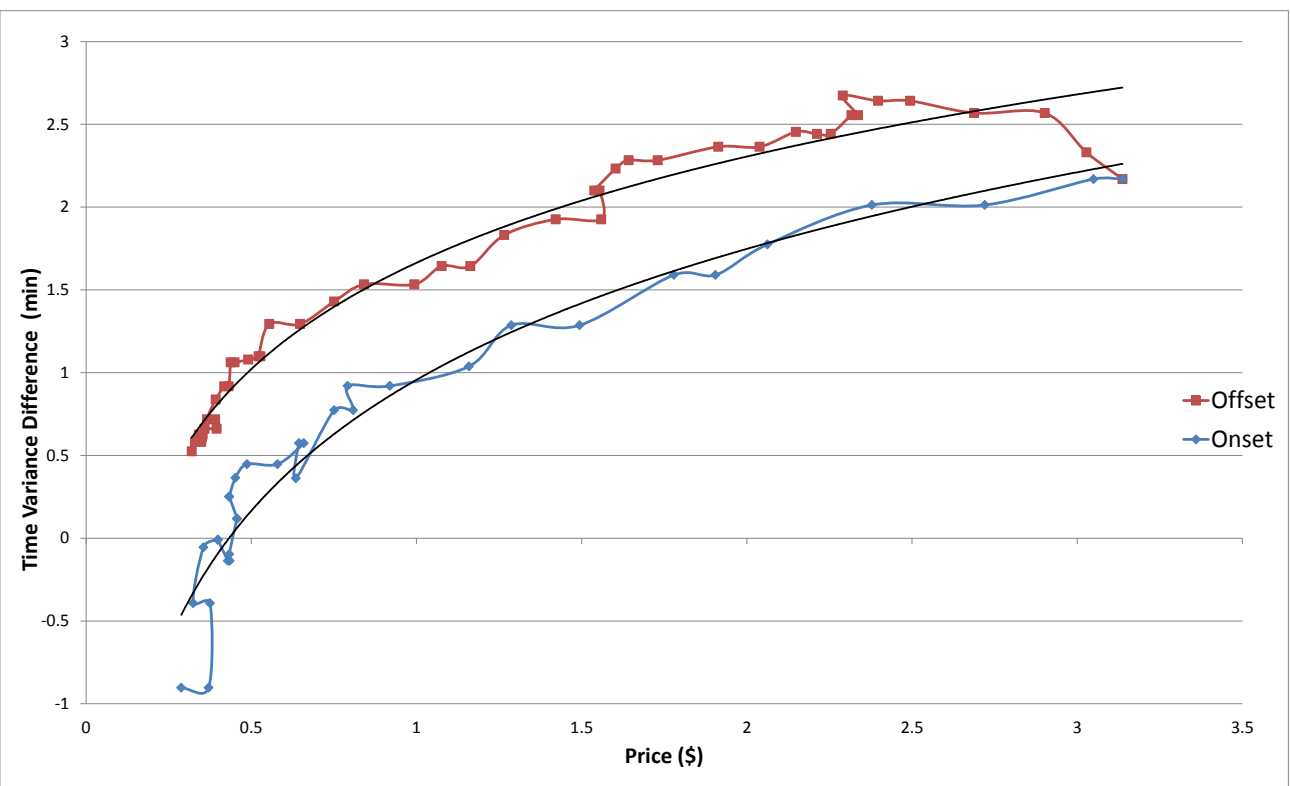

$\Delta V_{\text {onset }}=1.1413 \ln (P)+0.9566\left(r^{2}=0.942\right)$

$\Delta V_{\text {off set }}=0.926 \ln (P)+1.6636\left(r^{2}=0.9657\right)$

where $\Delta V$ is time variance difference in minutes and $P$ is price in USD 


\subsection{Calibration Process}

The flowchart (Figure 3) displays the lane choice model calibration cycle using the grid search technique. Once lane choice decisions for all vehicles have been completed, the percentage of vehicles using the HOT lane $(\operatorname{Pr}(L \mid R))$ is compared to the calibration target of $68.1 \%$. The model coefficients are then adjusted to increase or decrease HOT lane share and the process is repeated until the optimal coefficients are found.

Figure 3: Calibration Cycle

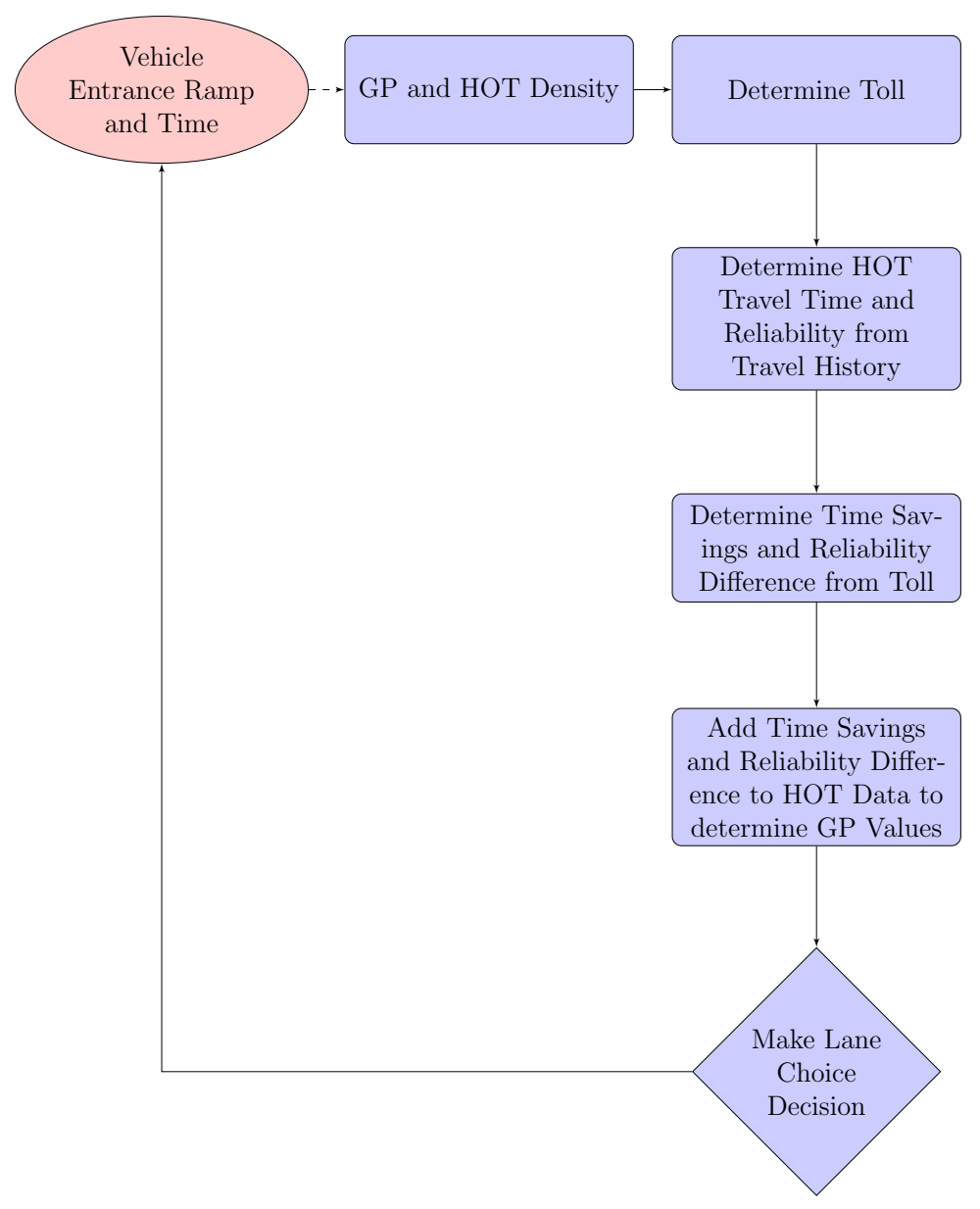

The lane choice parameters were for both congestion onset and offset. The resulting values are found in Table 3. 
Table 3: Lane Choice Model Parameters for Calibration

\begin{tabular}{cccc}
\hline Parameter & Carrion(2010) & Onset & Offset \\
\hline Expected Travel Time & -0.672 & -7.27 & -10.7 \\
Travel Time Variability & -0.228 & -2.47 & -3.63 \\
HOT Lane Toll & -6.94 & -6.94 & -6.94 \\
Alternative Specific Constant & -2.23 & 0 & 0 \\
\hline
\end{tabular}

\section{Alternative Pricing Scenarios}

Results from the lane choice model are used to test four alternative pricing strategies using a fixed demand partial equilibrium analysis.

Four HOT lane pricing strategies are tested that could serve as alternatives to the previous MnPASS pricing system. The previous system relied on a series of density and price tables to determine the toll based strictly on HOT lane density. The proposed alternatives determine the toll based on a mathematical function relating HOT lane density (and general purpose lane density in three of the strategies) to price. The three value pricing strategies use the difference in GP and HOT lane density to determine the toll. Due to the nonlinear relationship between density and time savings, two of the strategies are weighted by either HOT density or GP density.

The continuous function is similar to the previous pricing algorithm in that it relies strictly on HOT lane density for determining price; however, instead of relying on a series of tables, price is determined from a mathematical equation. The three other value pricing strategies incorporate GP lane density and use the difference in density between the HOT and GP lanes to determine price. Details of the pricing strategies are outlined below.

In all cases, the prices are confined to several constraints to match the existing pricing algorithm. The minimum price is $\$ 0.25$, the maximum $\$ 8.00$ and all prices are rounded to the nearest $\$ 0.25$. The following equation represents the constraints which are applied after the unconstrained price is determined.

$$
P_{\text {constrained }}=\operatorname{Rnd}\left(\operatorname{Min}\left(\operatorname{Max}\left(P_{\text {unconstrained }}, 0.25\right), 8.00\right), 0.25\right)
$$

$P_{\text {unconstrained }}$ may be defined several ways, as discussed below. 
Continuous Function Prices using this function are determined by:

$$
P_{\text {continuous }}=\alpha * K_{H O T}^{\beta}
$$

where $\mathrm{P}$ represents the price in USD and $\mathrm{K}$ the density in vehicles/mile/lane.

$K_{H O T}$ is found using the same method as the current algorithm (maximum downstream density averaged over last 6 minutes). $\alpha$ and $\beta$ are constants which can be adjusted to achieve the desired curve.

Unweighted Value Pricing While the current pricing algorithm only evaluates the density in the HOT lane, this pricing strategy computes price based on the difference in density between the GP and HOT lanes. The difference in density between the lane groups is correlated with a difference in time savings and therefore, the value provided by the HOT lane. Implementation of this pricing scheme (and subsequent strategies), will require the integration of GP density as a factor in determining price. GP density is averaged among parallel detectors. The maximum downstream GP density is then used to determine price, along with the maximum downstream HOT density.

$$
P_{\text {Value }_{\text {unweighted }}}=\gamma *\left[K_{G P}-K_{H O T}\right]
$$

$H O T_{\text {weighted }}$ Value Pricing Differences in density between GP and HOT lanes do not correlate directly to travel speeds. Rather, there is a correlation with the magnitude of densities. For example, little speed difference exists between 10 and 20 vehicles/mi/ln (6 and $12 \mathrm{veh} / \mathrm{km} / \mathrm{ln})$, both likely experience free flow speeds. However, a greater speed difference exists at higher densities (between 40 and $50 \mathrm{veh} / \mathrm{mi} / \mathrm{ln}$ (approximately 25 and 31 veh $/ \mathrm{km} / \mathrm{ln})$ ). This function weights the difference based on the magnitude of the HOT lane density. Price increases proportionally with HOT density.

$$
P_{\text {ValueHOT }_{\text {weighted }}}=\delta *\left[K_{G P}-K_{H O T}\right] * K_{H O T}
$$

$G P_{\text {weighted }}$ Value Pricing This pricing strategy is weighted based on GP density instead of HOT density. If $K_{G P}$ is much greater than $K_{H O T}$ and $K_{H O T}$ is very low, then the HOT weighted value pricing strategy would yield a low price even though there would be a significant value in using the HOT lane. By weighting based on $K_{G P}$, this strategy ties price more directly to the GP lane congestion and the actual time savings gained by using the HOT lane.

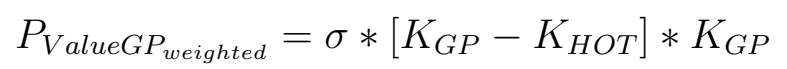


The $H O T_{\text {weighted }}$ strategy combines the value pricing concept with the current algorithm's direct correlation between HOT density and price. For this reason, we posit that this pricing strategy would provide the greatest improvement over the current pricing system while still maintaining some of the same logic. The continuous function, on the other hand, most closely resembles the previous pricing system (and was subsequently implemented), but does not account for the density in the GP lanes.

\subsection{Evaluation}

The calibrated HOT lane choice model is used to test the behavior of the alternative pricing strategies and how changing prices affect the share of transponder owning SOVs that use the MnPASS lane.

Each pricing strategy's coefficients were incrementally adjusted and the process rerun to determine the resulting HOT lane share $(\operatorname{Pr}(L \mid R))$. The average price and share were recorded for each iteration. The results were graphed and fit for each pricing strategy (congestion onset and offset).

Table 4: Pricing Strategy Coefficients for Constant Time Savings Lane Choice Model

\begin{tabular}{ccccc}
\hline Pricing Strategy & Iteration & Coefficient & Lane Share & Average Price \\
\hline $\begin{array}{c}\text { Continuous Function Pricing }(\alpha): \\
\text { (where } \beta=1.156)\end{array}$ & 1 & 0.059 & $78.3 \%$ & $\$ 1.77$ \\
\hline Unweighted Value Pricing $(\gamma):$ & 1 & 0.085 & $68.1 \%$ & $\$ 2.39$ \\
\hline$H O T_{\text {Weighted }}$ Value Pricing $(\delta):$ & 1 & 0.058 & $65.8 \%$ & $\$ 2.16$ \\
& 2 & 0.0033 & $68.1 \%$ & $\$ 1.87$ \\
\hline$G P_{\text {Weighted }}$ Value Pricing $(\sigma):$ & 1 & 0.0017 & $58.3 \%$ & $\$ 3.45$ \\
& 2 & 0.00089 & $68.1 \%$ & $\$ 2.28$ \\
\hline
\end{tabular}

Each line represents an iteration

The first iteration uses parameters to best match the previous algorithm based on average demand data from 2011 and 2012

The second iteration adjusts parameters to best match the HOT lane share calibration target of $68.1 \%$

Table 5 displays the regression results from fitting the Continuous pricing strategy using a first, second, third and fourth order polynomial function. The fourth degree polynomial functions for each scenario are displayed in Table 6 and graphs of the Continuous Function (congestion onset and offset) are displayed in Figure 4. 
Table 5: HOT Lane Share as a function of Price: Continuous Pricing, Congestion Onset

\begin{tabular}{|c|c|c|c|c|c|c|c|}
\hline Variable & 1st order & 2nd order & & 3rd order & & 4 th order & \\
\hline Intercept & $\begin{array}{r}21.46 \text { *** } \\
(5.321)\end{array}$ & $\begin{array}{r}-0.9280 \\
(3.140)\end{array}$ & & $\begin{array}{l}-15.297 \\
(1.997)\end{array}$ & $* * *$ & $\begin{array}{c}-25.884 \\
(0.9949)\end{array}$ & $* * *$ \\
\hline$P$ & $\begin{array}{r}1.017 \\
(1.291)\end{array}$ & $\begin{array}{r}35.019 \\
(2.892)\end{array}$ & $* * *$ & $\begin{array}{r}71.931 \\
(3.546)\end{array}$ & $* * *$ & $\begin{array}{l}108.91 \\
(2.670)\end{array}$ & $* * *$ \\
\hline$P^{2}$ & - & $\begin{array}{r}-4.5576 \\
(0.3788)\end{array}$ & $* * *$ & $\begin{array}{r}-17.218 \\
(1.129)\end{array}$ & $* * *$ & $\begin{array}{r}-40.396 \\
(1.516)\end{array}$ & $* * *$ \\
\hline$P^{3}$ & - & - & & $\begin{array}{r}1.0781 \\
(0.09485)\end{array}$ & $* * *$ & $\begin{array}{r}5.7446 \\
(0.2956)\end{array}$ & $* * *$ \\
\hline$P^{4}$ & $\begin{array}{l}- \\
-\end{array}$ & _ & & - & & $\begin{array}{r}-0.2941 \\
(0.01849)\end{array}$ & $* * *$ \\
\hline$n$ & 44 & 44 & & 44 & & 44 & \\
\hline$r^{2}$ & 0.0146 & 0.7825 & & 0.9486 & & 0.9931 & \\
\hline
\end{tabular}

(Standard error in parentheses)

Significance * 0.05, ** 0.01, *** 0.001

HOT lane share is dependent variable, $P$ is price in USD

Table 6: Pricing Function Model Equations

\begin{tabular}{lcc}
\hline Pricing Function & Model & $r^{2}$ \\
\hline \multicolumn{4}{c}{ Onset } \\
\hline Continuous & $\operatorname{Pr}(L \mid R)=-0.2941 P^{4}+5.7446 P^{3}-40.396 P^{2}+108.91 P-25.884$ & 0.9931 \\
Unweighted & $\operatorname{Pr}(L \mid R)=-0.1555 P^{4}+3.6124 P^{3}-31.812 P^{2}+106.14 P-26.573$ & 0.9909 \\
$H O T_{\text {weighted }}$ & $\operatorname{Pr}(L \mid R)=-0.3468 P^{4}+6.3111 P^{3}-40.349 P^{2}+98.579 P-22.116$ & 0.9493 \\
GP $P_{\text {weighted }}$ & $\operatorname{Pr}(L \mid R)=-0.1785 P^{4}+3.2471 P^{3}-22.649 P^{2}+66.301 P-13.515$ & 0.9604 \\
& \multicolumn{2}{c}{ Offset } \\
\hline Continuous & $\operatorname{Pr}(L \mid R)=-0.2394 P^{4}+4.4049 P^{3}-27.423 P^{2}+51.688 P+61.127$ & 0.9904 \\
UnweightedValue & $\operatorname{Pr}(L \mid R)=-0.1284 P^{4}+3.0066 P^{3}-23.308 P^{2}+51.99 P+61.762$ & 0.9887 \\
HOT $T_{\text {weighted }}$ & $\operatorname{Pr}(L \mid R)=-0.1652 P^{4}+2.9438 P^{3}-17.877 P^{2}+29.923 P+69.300$ & 0.9801 \\
GP $P_{\text {weighted }}$ & $\operatorname{Pr}(L \mid R)=-0.0546 P^{4}+1.2879 P^{3}-10.691 P^{2}+22.483 P+68.142$ & 0.9905 \\
\hline \multicolumn{4}{c}{$P$ is price in USD } &
\end{tabular}


Figure 4: Continuous Pricing Function

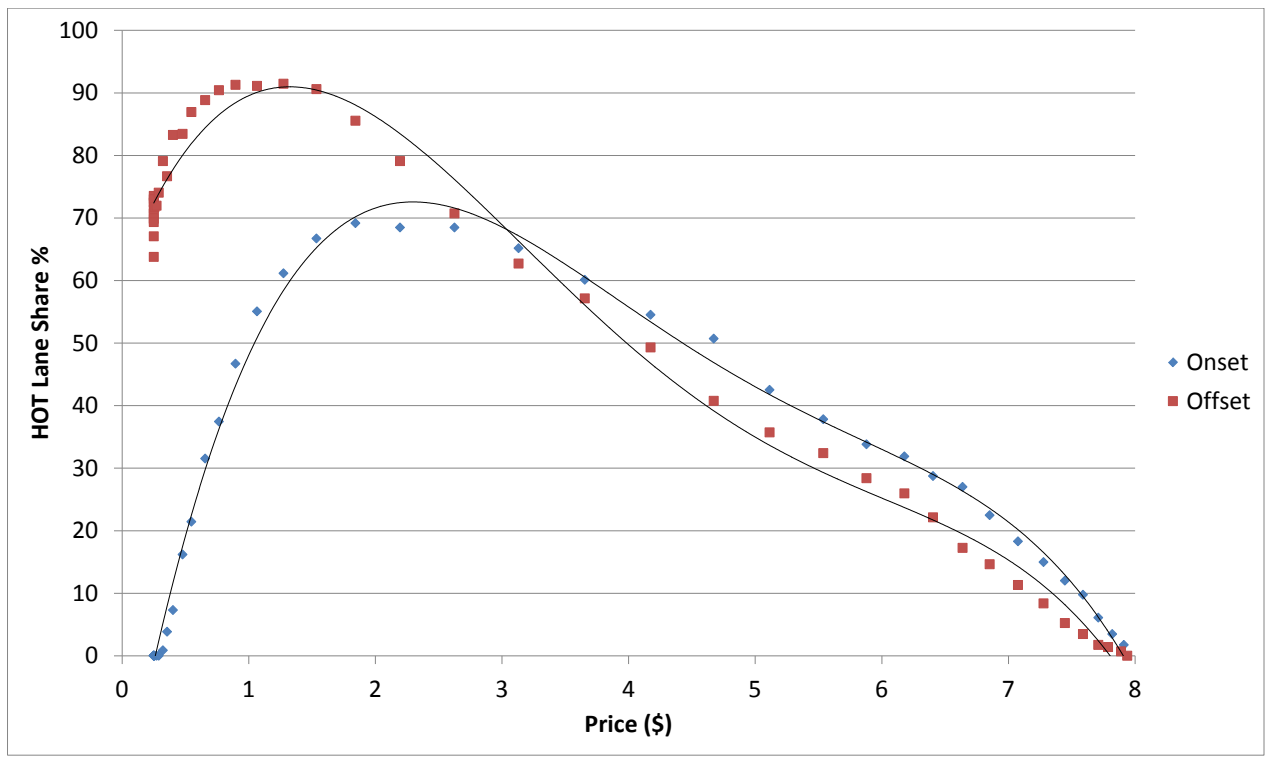

$P=\alpha * K_{H O T}^{\beta}$, where $\beta=1.156$ and $\alpha=0.065$

\subsection{Elasticity}

The functions above describe HOT lane share as a function of toll price. The elasticity of share to price is determined by taking the derivative of the function and multiplying by the quotient of price divided by share.

$$
\varepsilon_{P r(L \mid R)(P)}=\frac{P * \operatorname{Pr}(L \mid R)^{\prime}(P)}{\operatorname{Pr}(L \mid R)(P)}=\frac{d \ln P r(L \mid R)(P)}{d \ln P}
$$

Table 7: Average Elasticity Values (Fixed Time Savings)

\begin{tabular}{cc}
\hline Continuous Pricing & -0.372 \\
Unweighted Value Pricing & -0.260 \\
HOT Weighted Value Pricing & -0.175 \\
GP Weighted Value Pricing & -0.496 \\
\hline
\end{tabular}

Figure 5 graphs elasticity as a function of price for the continuous function pricing strategy (onset and offset). The elasticity equations are displayed below the figure. 
Figure 5: Continuous Pricing Function

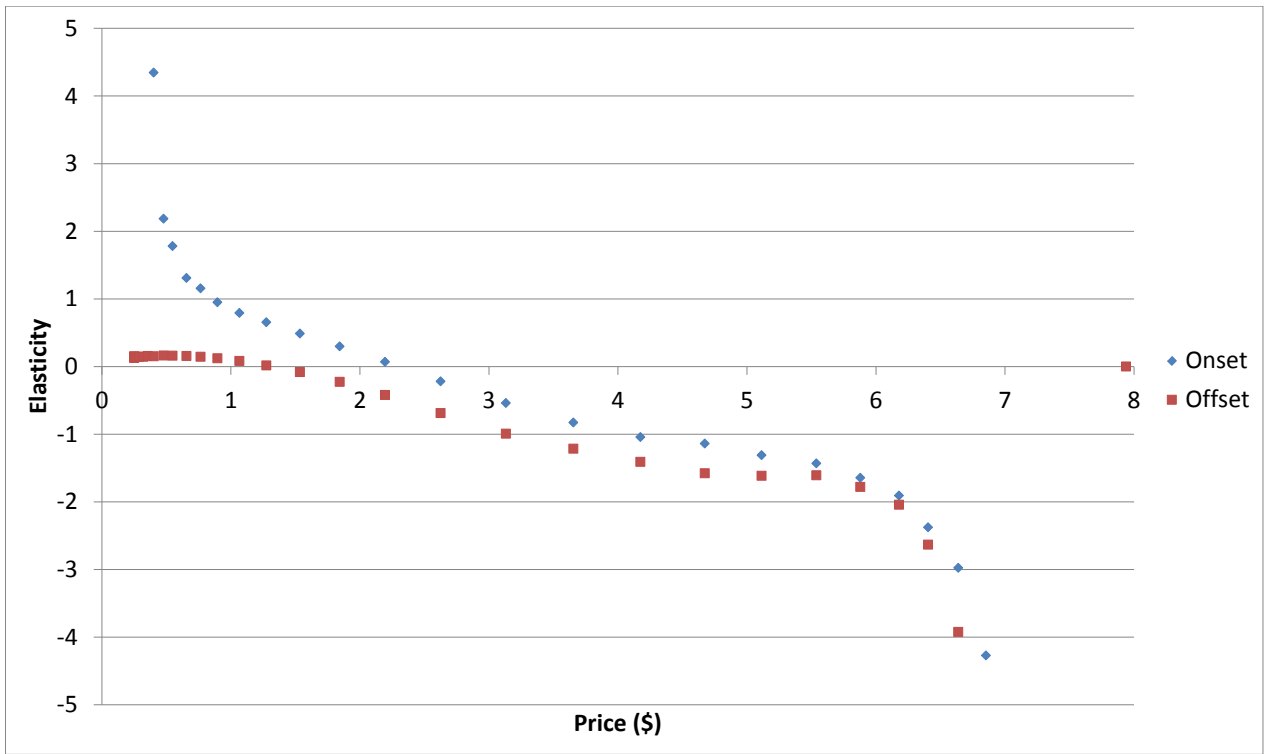

$\varepsilon_{P r(L \mid R)(P)}=\frac{P *\left(-1.176 * 4 * P^{3}+17.23 * P^{2}-80.79 * P+108.9\right)}{\operatorname{Pr}(L \mid R)(P)}$

$\varepsilon_{\operatorname{Pr}(L \mid R)(P)}=\frac{P *\left(-0.9576 * P^{3}+13.21 * P^{2}-54.85 * P+51.69\right)}{\operatorname{Pr}(L \mid R)(P)}$ where $p$ is price in USD

\subsection{Discussion}

All four pricing strategies show a similar pattern in the relationship between HOT lane share and price. The maximum HOT lane share during congestion onset is achieved between $\$ 2$ and $\$ 3$, whereas during congestion offset, the greatest HOT lane share occurs between $\$ 1$ and $\$ 2$. In general, the HOT lane share during congestion offset is greater than during the onset due to the greater time savings and reliability per dollar of toll as demonstrated previously in Figures 1 and 2. Table 8 shows the average price and HOT lane share for each pricing strategy along with the standard deviation.

Table 8: Average HOT lane share and Prices

\begin{tabular}{|c|c|c|c|c|c|c|}
\hline \multirow[t]{2}{*}{ Pricing Strategy } & \multirow[t]{2}{*}{ Avg Price $(\$)$} & \multirow[t]{2}{*}{ Std Dev Price $(\$)$} & \multicolumn{2}{|c|}{ Avg HOT lane share (\%) } & \multicolumn{2}{|c|}{ Std Dev HOT lane share $(\%)$} \\
\hline & & & Onset & Offset & Onset & Offset \\
\hline Continuous & 2.93 & 2.93 & 54.6 & 24.4 & 31.6 & 24.7 \\
\hline Unweighted & 3.19 & 3.20 & 54.1 & 24.5 & 36.9 & 32.6 \\
\hline HOT Weighted & 3.65 & 2.93 & 49.4 & 24.5 & 31.6 & 19.3 \\
\hline GP Weighted & 3.83 & 3.26 & 45.5 & 18.9 & 34.0 & 21.0 \\
\hline
\end{tabular}


Figure 4 shows the rise and fall of the HOT lane share as the toll increases (and therefore, time savings). When HOT lane share reaches its maximum, elasticity switches from positive to negative. Figure 6 outlines how changes in toll and time savings affect HOT lane share and ultimately, elasticity to price.

Figure 6: Toll and Time Savings Effect on HOT lane share

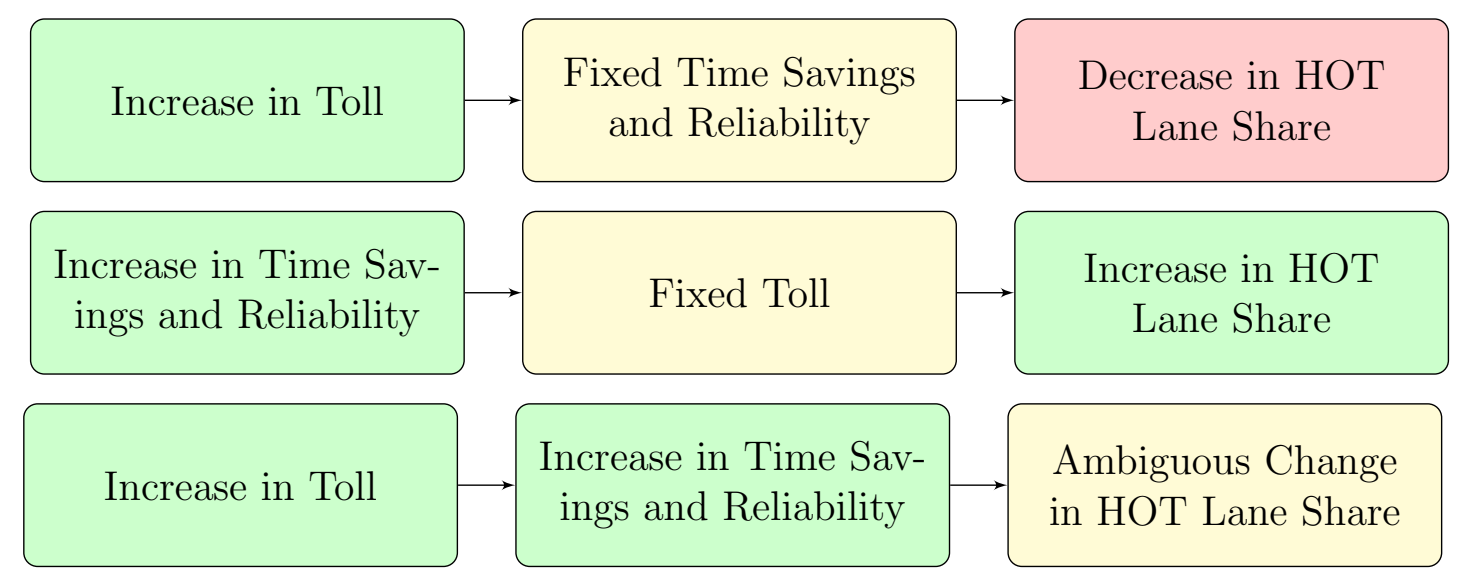

At lower tolls, an increase in price results in a higher HOT lane share (positive elasticity), whereas at higher tolls, an increase in price causes a decrease in HOT lane share (negative elasticity). At lower tolls, the improved time savings and reliability outweigh the increase in toll. However, at higher tolls, the increase in toll outweighs greater time savings and reliability causing the HOT lane share to decrease.

Based on an earlier version of this research, MnDOT selected the continuous function to replace the toll table described in Table 1.

\section{Conclusion}

This research estimated and calibrated a lane choice model for HOT lanes. In the model, simulated vehicles made decisions on whether to use the MnPASS lane based on the toll and their anticipated time savings and improved travel time reliability. Four alternative pricing scenarios were tested. The lane choice was determined at various price increments for each pricing system. These were plotted and fit with a polynomial, the derivatives of which correlate to the elasticity to price. In all cases, demand elasticity to price was positive at lower tolls and negative at higher tolls. MnPASS users recognize the correlation between the toll price and the time savings and travel time reliability provided by the lanes. The toll price acts as a proxy of downstream congestion. At lower tolls, the travel time savings and reliability advantages outweigh the cost of the toll and share of HOT lane users rises. However, at higher tolls, the cost of using the lane begins to outweigh the benefit and the 
share drops.

These results are estimated on a system where drivers have incomplete information about travel time savings from HOT lane usage, and use price as a signal of time savings. In a context where drivers were better informed (e.g. through Variable Message Signs or real-time congestion-aware GPS navigation systems), results would likely be significantly different.

Future research should field test alternative pricing strategies and parameter values to identify which best achieves the goals of maximizing use of the HOT lanes while maintaining reliable free-flowing speeds, recognizing that travelers may change their sensitivity to price if the perceived relationship between price and travel time savings changes.

The value of HOT lanes in providing free-flow travel conditions for those willing to pay, can be combined with the opportunity to use these lanes for freeway-based Bus Rapid Transit (express buses) now, and as a separate restricted network for Automated Vehicles in the future.

\section{Bibliography}

Buckeye, K. (2012). Performance evaluation of I-394 MnPASS express lanes in minnesota. Transportation Research Record: Journal of the Transportation Research Board (2278), $153-162$.

Cao, X. J., L. Munnich, H. Liu, X. S. He, Z. Xu, and Y. A. Huang (2012). Benefit and cost analysis of the I-394 MnPASS program.

Carrion, C. (2010). Value of Reliability: Actual Commute Experience Revealed Preference Approach. Ph. D. thesis, University of Minnesota.

Carrion, C. and D. Levinson (2012). Value of travel time reliability: A review of current evidence. Transportation research part A: policy and practice 46(4), 720-741.

Dahlgren, J. (1998). High occupancy vehicle lanes: Not always more effective than general purpose lanes. Transportation Research Part A: Policy and Practice 32(2), 99-114.

Dahlgren, J. (2002). High-occupancy/toll lanes: where should they be implemented? Transportation Research Part A: Policy and Practice 36 (3), 239-255.

Dupuit, J. (1844). On the measurement of the utility of public works. International Economic Papers 2(1952), 83-110.

Gardner, L., S. Boyles, H. Bar-Gera, and K. Tang (2014). Robust tolling schemes for highoccupancy toll facilities under variable demand. Transportation Research Record: Journal of the Transportation Research Board (2450), 152-162. 
Goodall, N. and B. Smith (2010). What drives decisions of single-occupant travelers in highoccupancy vehicle lanes? investigation using archived traffic and tolling data from mnpass express lanes. Transportation Research Record: Journal of the Transportation Research Board (2178), 156-161.

Halvorson, R. and K. R. Buckeye (2006). High-occupancy toll lane innovations: I-394 MnPASS. Public Works Management \& Policy 10(3), 242-255.

Harlow, T. (2015). The drive: MnDOT to tweak MnPass pricing. Star Tribune November 9, 2015.

Janson, M. and D. Levinson (2014). HOT or not: Driver elasticity to price on the MnPASS HOT lanes. Research in Transportation Economics 44, 21-32.

Munnich, L. and K. Buckeye (2007). I-394 MnPASS High-Occupancy Toll Lanes: Planning and Operational Issues and Outcomes (Lessons Learned in Year 1). Transportation Research Record: Journal of the Transportation Research Board 1996(1), 49-57.

Owen, A., M. Janson, and D. Levinson (2014). Incremental accessibility benefits and choice of subscriptions for high-occupancy toll lanes. Transportation Research Record: Journal of the Transportation Research Board (2412), 93-99.

Pahaut, S. and C. Sikow (2006). History of thought and prospects for road pricing. Transport policy $13(2), 173-176$.

Small, K. A., C. Winston, and C. A. Evans (2012). Road work: A new highway pricing and investment policy. Brookings Institution Press.

Tilahun, N. and D. Levinson (2009). Unexpected delay and the cost of lateness on i-394 high occupancy/toll lanes. Travel Demand Management and Road User Pricing: Success, Failure and Feasibility, 173-184.

Vickrey, W. S. (1969). Congestion theory and transport investment. The American Economic Review 59(2), 251-260.

Yang, H. and H.-J. Huang (2005). Mathematical and economic theory of road pricing. Elsevier. 